\title{
Repercussões do papel de cuidador nas atividades de lazer de cuidadores informais de idosos dependentes
}

\section{Repercussions of the role of carer in the leisure activities of informal caregivers's dependent elder}

\author{
Marina Picazzio Perez Batista ${ }^{1}$, Fernanda Luppino Miccas ${ }^{2}$, \\ Fernanda de Sousa Forattore ${ }^{3}$, Maria Helena Morgani de Almeida ${ }^{4}$, \\ Tatiana Vieira do Couto ${ }^{5}$
}

\begin{abstract}
BATISTA, M. P. P.; MICCAS, F. L.; FORATTORE, F. S.; ALMEIDA, M. H. M.; COUTO, T. V. Repercussões do papel de cuidador nas atividades de lazer de cuidadores informais de idosos dependentes. Rev. Ter. Ocup. Univ. São Paulo, v. 23, n. 2, p. 186-192, maio/ago. 2012.

RESUMO: A responsabilidade pelo cuidado acarreta transformação no cotidiano dos cuidadores. Frente às obrigações da vida cotidiana, que causam desgaste físico e emocional, as atividades de lazer permitem o restabelecimento do indivíduo, além de propiciar desenvolvimento pessoal e social. Objetivo: O presente estudo buscou identificar, junto a cuidadores informais de idosos dependentes internados na enfermaria de Geriatria do Hospital do Servidor Público Estadual, suas atividades de lazer e fatores que influenciam na realização das mesmas. Metodologia: Os dados do estudo foram coletados por meio de entrevista semi-estruturada e analisados pelo método de análise de conteúdo. As variáveis quantitativas foram descritas por meio de frequência simples. Resultado: Encontrou-se que a maioria dos cuidadores entrevistados diminuíram a quantidade e frequência na realização de atividades de lazer por assumirem a responsabilidade de cuidado com o idoso dependente. Vinculado à esta responsabilidade, os cuidadores mencionaram a hospitalização e aspectos emocionais como fatores que influenciam na execução destas atividades. Conclusão: Por meio do impacto observado nas atividades de lazer dos cuidadores, este estudo possibilita a reflexão sobre um tema de grande relevância: o cuidado ao cuidador.
\end{abstract}

DESCRITORES: Atividades de lazer/psicologia; Idoso; Cuidadores/psicologia; Atividades cotidianas/psicologia.

\footnotetext{
1. Terapeuta Ocupacional. Especialização em Atendimento Interdisciplinar em Geriatria e Gerontologia pelo Hospital do Servidor Público Estadual. Universidade de São Paulo, Faculdade de Medicina.

2. Terapeuta Ocupacional. Especialização em Atendimento Interdisciplinar em Geriatria e Gerontologia pelo Hospital do Servidor Público Estadual. Hospital do Servidor Público Estadual Francisco Morato de Oliveira.

3. Terapeuta Ocupacional. Especialização em Atendimento Interdisciplinar em Geriatria e Gerontologia pelo Hospital do Servidor Público Estadual. Hospital do Servidor Público Estadual Francisco Morato de Oliveira.

4. Profa. Dra. do Curso de Terapia Ocupacional da Faculdade de Medicina da Universidade de São Paulo.

5. Terapeuta Ocupacional. Preceptora do Aprimoramento em Atendimento Interdisciplinar em Geriatria e Gerontologia pelo Hospital do Servidor Público Estadual. Hospital do Servidor Público Estadual Francisco Morato de Oliveira.

Endereço para correspondência: Rua Cipotânea, 51. Cidade Universitária. CEP: 05360-000 - São Paulo, SP. E-mail: marinapperez@, yahoo.com.br
} 


\section{INTRODUÇÃO}

$\mathrm{O}$ envelhecimento da população mundial é considerado um dos fenômenos de maior impacto no início deste século. Em decorrência deste processo, aumentou a prevalência e incidência de doenças crônico-degenerativas, que causam incapacidades (LITVOC; BRITO, 2004). As incapacidades crônicas acarretam perda de independência gerando dificuldades ou impossibilidades de realização das atividades de vida diária, influenciando a capacidade funcional dos idosos (KARSCH; LEAL, 1998).

$\mathrm{O}$ conceito de capacidade funcional refere-se à capacidade de o indivíduo manter suas habilidades físicas e mentais requeridas para uma vida independente e autônoma (BRASIL, 1999). Há diversos instrumentos de avaliação para aferir a capacidade funcional. Um deles é a Medida de Independência Funcional (MIF), que avalia o nível de comprometimento funcional de um indivíduo para a realização de atividades de vida diária e a quantidade de auxílio requerido para desempenho destas atividades (RIBERTO et al., 2004).

O conjunto de atividades que compõe a MIF pode ser dividido nos domínios motor e cognitivo, que determinam as subdivisões do instrumento: MIF motora e MIF cognitiva/ social. O domínio motor é composto de treze atividades, subdivididas em quatro eixos: cuidados pessoais, controle de esfíncter, transferência/mobilidade e locomoção. O domínio cognitivo é composto por cinco atividades, subdivididos em dois eixos: comunicação e cognição social. Na avaliação de cada uma das atividades, de ambos os domínios, é possível se obter uma pontuação que pode variar de 1 (dependência total) a 7 (independência completa) (RIBERTO et al., 2004).

A dificuldade ou impossibilidade de realização das atividades de vida diária de forma independente, faz com que o idoso demande auxílio para executá-las. Este auxílio pode se dar por meio do uso de equipamentos de tecnologia assistiva, mudanças ambientais e comportamentais ou necessidade de ajuda de outrem (RIBERTO et al., 2004).

Quando o idoso requer a ajuda de outra pessoa, o cuidador se torna o principal agente de suporte para o cuidado (KARSCH; LEAL, 1998; CALDAS, 1998). O cuidador pode ser identificado como formal, quando é um profissional contratado para assumir a tarefa do cuidar, ou informal, quando é um familiar, amigo e/ou voluntário da comunidade que assume a responsabilidade pelo cuidado (CALDAS, 1998).

Os cuidadores informais podem ser classificados em primários e secundários. Os cuidadores primários são os principais responsáveis pelo cuidado do idoso, realizando portanto, a maioria das tarefas envolvidas no ato de cuidar.
Os cuidadores secundários não possuem o mesmo nível de responsabilidade e decisão quando comparado aos primários. Frequentemente os cuidadores secundários atuam revezando o cuidado com os primários auxiliando, de forma pontual, nas atividades básicas de vida diária (NERI; CARVALHO, 2004).

O exercício do papel de cuidador pode trazer um impacto negativo sobre a saúde emocional e física do cuidador. As situações, nas quais ocorre algum tipo de sobrecarga, podem resultar em redução no nível de imunidade, aumento da susceptibilidade a enfermidades, depressão e sintomas de estresse (NERI; CARVALHO, 2004).

Ras Vidal et al. (2006) conduziram estudo com cuidadores de idosos com doenças crônicas, no qual $90 \%$ referiram que suas vidas foram modificadas depois de assumirem o papel de cuidador. Revelou também que a responsabilidade pelo cuidado acarretou aos cuidadores problemas psíquicos $(52,3 \%)$, físicos $(50,8 \%)$ e nas relações sociais $(56,8 \%)$. Outra pesquisa estudou a associação entre a sobrecarga física e emocional implicada na responsabilidade do cuidado às mudanças na saúde dos cuidadores, e identificou: cansaço, perda de peso, preocupação e aparecimento de sintomas e doenças. Além disso, mostrou que a sobrecarga acarretava modificações na auto-estima dos cuidadores e transformações em seu cotidiano (RODRIGUES et al., 2006).

Em relação às mudanças no cotidiano, identifica-se que os cuidadores tendem a reduzir seus contatos sociais; apresentam dificuldades financeiras advindas do gasto excessivo com o cuidado do idoso e reduzem o tempo para o cuidado consigo e para a realização de atividades de lazer (ROCHA et al., 2008)

Em relação às atividades de lazer, estas são definidas como atividades não-obrigatórias, de interesse e desejo do indivíduo, escolhidas livremente, e para as quais se sente motivado. Compreende-se que o prejuízo nestas atividades pode interferir negativamente na vida do cuidador, uma vez que as atividades de lazer propiciam o desenvolvimento pessoal e social dos indivíduos e permitem o restabelecimento físico e emocional decorrentes do desgaste acarretado pelas obrigações da vida cotidiana (REQUIXA, 1980).

Assim, considerando a relevância das atividades de lazer para o bem-estar dos indivíduos, o presente estudo se propôs a identificar, junto a cuidadores informais de idosos dependentes, as atividades de lazer e os fatores que influenciam na sua realização.

\section{METODOLOGIA}

O estudo foi realizado na Enfermaria de Geriatria do Hospital do Servidor Público Estadual "Francisco Morato 
de Oliveira" (HSPE- FMO). Trata-se de estudo descritivoexploratório, realizado de setembro a outubro de 2007, com cuidadores informais de idosos dependentes internados na enfermaria neste período. Os critérios para inclusão dos participantes no estudo foram: a) ser cuidador de idoso internado na enfermaria de Geriatria do HSPE-FMO; b) ser cuidador informal e não contar com auxílio de cuidador formal; c) ser cuidador de idoso dependente.

A fim de caracterizar os idosos da enfermaria quanto ao nível de dependência para a realização de atividades de vida diária, foi aplicada a Medida de Independência Funcional, em seu domínio motor. Os idosos que obtiveram a pontuação 1, foram classificados como dependentes para realização de atividades básicas de vida diária (RIBERTO et al., 2004).

Os cuidadores dos idosos dependentes e que atenderam aos demais critérios de inclusão foram convidados a participarem do estudo, por meio de assinatura do termo de consentimento livre e esclarecido.

Foram realizadas entrevistas semi-estruturadas com os cuidadores. As questões do roteiro de entrevista buscaram conhecer quais eram as atividades de lazer dos cuidadores e se o cuidado com o idoso influenciava em sua realização. Foram também formuladas perguntas que visaram a identificação dos cuidadores, no que diz respeito ao gênero, à idade, à escolarização e ao tempo de cuidado com o idoso totalmente dependente.

As entrevistas foram gravadas e transcritas. Como procedimento de interpretação dos dados coletados empregou-se a análise de conteúdo por categorias temáticas, nas quais foram selecionadas algumas falas para exemplificação dos temas observados. As variáveis quantitativas relacionadas à frequência de ocorrência dos temas foram descritas por meio de frequência simples (BARDIN, 1977). Para fim de preservação da identidade dos participantes, cada um foi identificado pela letra $\mathrm{E}$, seguida de número.

Este estudo foi aprovado pelo Comitê de Ética em Pesquisa do Hospital do Servidor Público Estadual sob protocolo $\mathrm{n}^{\mathrm{o}}$ 063/07, estando de acordo com a Resolução 196/196 do Conselho Nacional de Saúde.

\section{RESULTADOS E DISCUSSÃO}

\section{Perfil da população}

Participaram do estudo dez cuidadores de idosos dependentes internados na enfermaria.

Dentre a amostra, $90 \%$ são do sexo feminino, com idade entre 37 e 74 anos. Em relação ao grau de parentesco com o idoso, $70 \%$ são filhos e $30 \%$ são cônjuges. Os cuidadores, em sua maioria ( $80 \%$ ), residiam com o idoso e apresentavam média de dois anos e sete meses de cuidados com o idoso totalmente dependente. Este perfil encontrado nos cuidadores participantes correspondeu ao identificado em outros estudos (GÁLVEZ MORA et al., 2003; RAS VIDAL et al., 2006).

Em relação ao estado civil, $50 \%$ eram casados, $20 \%$ divorciados, $20 \%$ viúvos e $10 \%$ solteiros. Quanto ao nível de escolaridade, 40\% possuíam Ensino Fundamental, 20\% Ensino Médio e 20\% Superior.

Quanto à divisão dos cuidados com os demais membros da família, foi encontrado neste estudo que 50\% dos cuidadores entrevistados são os únicos responsáveis pelos cuidados. Muitos deles declararam não receber ajuda nos cuidados com o paciente, o que coincidiu com dados demonstrados na literatura (GÁLVEZ MORA et al., 2003; RODRIGUES et al., 2006). Ao mesmo tempo, identificou-se que dentre os cuidadores que dividem o cuidado com outro membro da família, $40 \%$ deles têm a maior responsabilidade sobre o paciente quando comparado aos demais. Desse modo, a maioria são cuidadores primários (NERI; CARVALHO, 2004).

\section{Atividades de lazer e fatores que influenciam em sua realização}

Em relação à realização de atividades de lazer, $100 \%$ dos participantes realizavam pelo menos uma atividade antes de assumirem o papel de cuidador. Dentre as atividades citadas pelos cuidadores foram mencionadas: participar de atividades comunitárias, passear, viajar, realizar trabalho voluntário, estudar, praticar atividades físicas e visitar familiares e amigos.

Metade dos entrevistados deixaram de realizar pelo menos duas atividades de lazer, como ilustram os relatos:

" Hoje eu não passeio mais. Faz cinco anos que eu tô presa dentro de casa sem sair. Porque eu não tenho coragem de sair e deixar minha mãe" (E3)

Ainda, verificou-se que os cuidadores que continuaram realizando atividades de lazer após assumirem a tarefa de cuidar, diminuíram o número destas atividades e sua frequência de realização quando comparado à momentos anteriores de sua vida.

"agora com esse problema da minha mãe a gente tem que estar mais disponivel. Eu ainda costuro um pouco, mas não é como era antigamente, que eu costurava muito" (E7)

Um quinto dos cuidadores (20\%) não deixou de 
realizar nenhuma atividade de lazer, porém destes, metade relatam que a realização destas atividades ficou cada vez mais raro. Estes dados encontram correspondência com um estudo sobre cuidadores de idosos crônicos, no qual a maioria dos participantes também referiu que não tem tempo livre para realização de atividades que não se relacionam com seu papel de cuidador (RAS VIDAL et al., 2006).

Dentre os participantes, $30 \%$ ao assumirem o papel de cuidadores deixaram de realizar todas as suas atividades de lazer conforme relatos abaixo:

“depois que ele piorou eu larguei de tudo." (E5)

"A minha vida é essa, não tenho nada mais, lazer nenhum(...) fico direto, é 24 horas no ar com ela." (E2)

Dos cuidadores entrevistados, $30 \%$ consideram o cuidado com o paciente como atividade de lazer, sendo que $66 \%$ destes citaram esta como a única atividade de lazer que realizam atualmente:

"A minha diversão é essa, meu lazer, cuidar dela." (E6)

Dados encontrados na literatura mostram que cuidadores referem aspectos positivos relacionados ao papel que exercem tais como sentirem-se úteis e necessários, referido por $76 \%$ dos cuidadores, e sentir-se bem consigo mesmo e com a vida, apontado por 70\% (WOLFF, 2007). Como identificado no relato:

"Eu tenho prazer de cuidar dele porque a gente viveu a vida toda juntos." (E10)

Como pôde ser percebido pelos relatos apresentados, a necessidade de se assumir a responsabilidade pelo cuidado do idoso dependente é um aspecto que influencia na realização de atividades de lazer para quase a totalidade dos entrevistados.

Os cuidadores mencionaram alguns aspectos que influenciam na possibilidade de realização de atividades de lazer. A hospitalização foi referida como um destes fatores. Dentre os entrevistados que mencionaram este aspecto, $22 \%$ referem que há um impacto negativo da hospitalização na realização destas atividades.

"não tô fazendo mais nada. Fico só aqui (...) é só isso aí agora." (E1)

Contrariamente $22 \%$ relacionaram a hospitalização do idoso como fator que traz a possibilidade de retomada, ainda que momentânea, das atividades de lazer. Isto porque o fato do idoso estar hospitalizado permite ao cuidador maior tempo disponível para o cuidado consigo, diferentemente de quando o idoso está no domicílio, onde o cuidador necessita dispender de seu tempo integral para o cuidado:

"Eu gosto muito de fazer crochê(...) Aqui mesmo, no hospital, eu sento e faço (...)Em casa é mais dificil de fazer porque em casa eu tenho que tomar conta dela” (E4)

Outros fatores identificados como negativos na realização de atividades de lazer dos cuidadores foram o sentimento de preocupação constante do cuidador com o idoso, o sentimento de culpa e a anedonia. Tais aspectos foram referidos por $90 \%$ dos entrevistados.

\footnotetext{
“(...)se eu saio pra alguma coisa, por exemplo vou fazer uma visita, eu não tô relaxada. Tô preocupada, com a cabeça ligada que ele tá precisando de mim." (E8)

"eu vou numa festa e não consigo aproveitar, parece que você se culpa né. Você fala: ai, eu não devia tar aqui! será que quem tá lá vai cuidar? (...)você se anula e se culpa e quando você pode ir você não aproveita. E é de não ter aquele peso, eu tô aqui me divertindo e tem uma pessoa lá sofrendo." (E3)

"é dificil fazer as coisas hoje que dá prazer...nada dá prazer" (E2)
}

O impacto psicológico do cuidado nos cuidadores de idosos, encontrado neste estudo, também é explicitado em outros trabalhos. Estes estudos observaram que 71,4\% dos cuidadores principais se encontravam em situação de sofrimento psíquico, e referem um conjunto de sintomas decorrentes desse processo: preocupação com o paciente, sinais depressivos e ansiedade (GÁLVEZ MORA et al., 2003; GRUNFELD et al., 2004; RODRIGUES et al., 2006).

Tilden et al. (2004) e Rocha et al. (2008) identificaram que a percepção do sofrimento do idoso é um fator associado ao alto nível de tensão dos cuidadores. Este aspecto também foi percebido em alguns relatos extraídos dos participantes deste estudo:

"101 anos acho que ela já tá com carga demais. A gente vê uma mãe sofrer na cama é triste(...)A gente fica assim desolada, né." (E10)

$40 \%$ dos cuidadores referiram como importante na realização de atividades de lazer, a divisão de cuidados com outros familiares: 
"Quando eu vou na Igreja quem fica com meus pais é meu esposo, e quando ele vai pescar eu fico. A gente troca." (E8)

"Se eu tivesse uma pessoa que me ajudasse a tomar conta da minha mãe, alguém que eu pudesse deixar com confiança, quem sabe eu até voltava pro clube, fazer esporte, caminhar, que eu gosto muito." (E5)

Estes dados encontram consonância com estudo que encontrou maior nível de tensão em cuidadores que assumem sozinhos a responsabilidade pelos cuidados de um doente crônico, quando comparados à cuidadores que dividem o cuidado (TILDEN et al., 2004).

Este estudo encontrou também que mais da metade dos cuidadores que diminuíram ou deixaram de realizar atividades de lazer (64\%), acreditam que, com a morte do idoso, tenham condições de realizá-las:

"Eu penso, se um dia Deus levar ela, eu penso em sair, viajar, passear” (E10)

Grande parte dos cuidadores (80\%) associou a condição de alta dependência do idoso à sua possibilidade de óbito iminente.

"Esse ano ela tá bem debilitada, não sei o que vai acontecer no aniversário dela. Eu gostaria que ela fizesse 91 anos. Sei lá.” (E2)

Importante ressaltar que pôde ser observado que para um dos cuidadores, a entrevista se constituiu como momento de reflexão para retomada de atividades de lazer:

"Mas voltar a ver os passarinhos ainda dá, no sábado e domingo ainda dá. Eu tinha até esquecido, sabia. (...) Mas pelo menos sentar pra ver os passarinhos eu vou começar" (E2)

\section{Outras modificações no cotidiano dos cuidadores}

Os cuidadores entrevistados apresentaram outras modificações em seu cotidiano advindas da responsabilidade do cuidar, não abordadas inicialmente no roteiro de entrevista. Alguns cuidadores mencionaram que as necessidades de cuidado os obrigaram a mudar para a casa do idoso e outros optaram por cuidar do idoso em sua própria residência:

"Depois que ela ficou doente eu fui morar com ela."

“Meus pais moram comigo há 5 anos, pela doença da minha mãe.” (E1)

Tal fato modificou a rotina destes cuidadores, tal como observado no relato:

"Nem durmo mais no meu quarto, durmo do lado dela, ponho meu colchão do ladinho dela" (E4)

Um dos entrevistados referiu ter sido necessário pedir afastamento de seu trabalho devido à necessidade de assumir o papel de cuidador.

"deixei de trabalhar simplesmente porque tenho que cuidar dela" (E6)

Esta experiência também foi relatada em um estudo no qual $5 \%$ dos cuidadores pararam de trabalhar ou diminuíram sua jornada de trabalho por ter que cuidar do paciente (GRUNFELD et al., 2004). Rocha et al. (2008) também apontam que é freqüente as interrupções na carreira profissional quando a pessoa precisa assumir o papel de cuidador.

Em outro relato pôde ser percebido que o cuidador necessitou responsabilizar-se por atividades que anteriormente eram realizadas pelo idoso:

"Tudo era ele, ele que resolvia tudo, mas depois que ele ficou doente quem faz sou eu.(...) eu que tenho que resolver tudo" (E3)

Outros cuidadores mencionaram diminuição das atividades de auto-cuidado:

"só tomo banho porque tenho que tomar, né. Tem dias que até o chuveiro eu olho assim e me dá paúra." (E10)

O presente estudo identificou o impacto no autocuidado dos entrevistados, o que também foi identificado por Rodrigues et al. (2006) e Wolff et al. (2007). Estes autores relatam que os cuidadores de idosos estão sempre presentes e disponíveis às necessidades do idoso que prestam assistência, muitas vezes descuidando de seu próprio bemestar (RODRIGUES et al., 2006; WOLFF et al., 2007). Muitos cuidadores dispendem maior tempo com o idoso do que consigo mesmo, alguns atribuindo a negligência de seu autocuidado à sobrecarga de tarefas e obrigações por ele exercida (RODRIGUES et al., 2006).

Quarenta por cento dos cuidadores identificaram a redução do convívio com outros familiares, como observado nos relatos a seguir: 
"ficar junto com a minha familia, meu marido e meus filhos, e isso ficou muito dificil com o estado da minha mãe." (E7)

Os relatos descritos acima confirmam os encontrados por Rocha et al. (2008) que mostram eu o cuidador informal tem maior tendência ao isolamento social e pouco tempo para manter contato com amigos e familiares.

\section{CONCLUSÃo}

De um modo geral, é possível encontrar estudos que demonstram o impacto do cuidar para os cuidadores em diversos âmbitos: físico, psicológico, social e ocupacional (RAS VIDAL et al., 2006; RODRIGUES et al., 2006). Neste estudo foi possível conhecer mais especificamente um dos aspectos importantes que compõe o cotidiano: a realização de atividades de lazer.

Observou-se que estas atividades foram negligenciadas ou diminuídas pela grande maioria dos cuidadores em decorrência do comprometimento com as responsabilidades advindas do cuidado com o idoso totalmente dependente. Nessa direção, mais da metade dos cuidadores relacionou a morte do idoso com a possibilidade de retomada de suas atividades de lazer, sendo possível inferir a grande influência do papel de cuidador na realização destas atividades.

Conforme Requixa (1980) as atividades de lazer permitem o desenvolvimento pessoal e social e recuperação psicossomática causado pelo desgaste das obrigações cotidianas. A partir dos resultados deste estudo, é possível inferir que os cuidadores são uma parcela da população que sofre prejuízo na vivência das atividades de lazer. Sugere-se que na atenção ao idoso dependente sejam consideradas as necessidades específicas de seus cuidadores, de modo que se busque oferecer suporte para prevenção do risco à sua saúde e para o impacto nas atividades de lazer advindo da grande responsabilidade do cuidado.

È relevante ressaltar que, apesar de nenhuma das questões do roteiro de entrevista terem abordado outras atividades do cotidiano dos cuidadores além de atividades de lazer, os entrevistados espontaneamente mencionaram outros aspectos da rotina que foram modificados pelo fato de assumirem os cuidados com o idoso, tais como: diminuição do autocuidado, do convívio social, afastamento do trabalho e mudanças relacionadas ao seu local de residência.

Esta pesquisa permite a reflexão acerca da relevância do impacto do cuidar nas atividades de lazer de cuidadores de idosos dependentes, porém por se tratar de estudo qualitativo, apresenta limitação quanto ao tamanho da amostra, que não torna possível a generalização dos resultados.

Outra limitação é que a amostra foi restrita à cuidadores informais de idosos, sendo excluídos aqueles que tinham auxílio de cuidadores formais. Acredita-se que contar com auxílio de cuidadores formais propicie aos cuidadores informais o exercício de suas atividades de lazer. Neste sentido, o estudo identificou que muitos cuidadores atribuíram a possibilidade de realizar atividades de lazer à divisão de cuidados com outros membros da família. Para futuros estudos, sugere-se comparar o impacto do cuidar nas atividades de lazer de cuidadores informais que recebem e que não recebem auxílio de cuidadores formais.

BATISTA, M. P. P.; MICCAS, F. L.; FORATTORE, F. S.; ALMEIDA, M. H. M.; COUTO, T. V. Repercussions of the role of carer in the leisure activities of informal caregivers's dependent elder. Rev. Ter. Ocup. Univ. São Paulo, v. 23, n. 2, p. 186-192, maio/ago. 2012.

ABSTRACT: The responsibility for caring brings transformations in the caregivers's daily lives. Opposing everyday life tasks, which result in both physical and emotional stress, leisure activities allow individual recovery as well as social and personal development. The aim of this study was to identify among the informal caregivers, who take care of dependent elders in the geriatric's ward at Hospital do Servidor Público Estadual, which their leisure activities are and the factors which affect their accomplishment. Data were collected through semi-structured interviews and analyzed by the use of the Content Analysis Method. The quantitative variables were described through simple frequency. As a result, it could be noticed that most of the interviewed caregivers decreased both quantity and frequency of their leisure activities due to their responsibilities in assisting dependent elders. Furthermore caregivers mentioned hospitalization and emotional aspects as factors that affected those activities. Therefore, through the impact observed in the caregiver's leisure activities, this study contributed to reflecting a very relevant theme: the care for the caregivers.

KEY WORDS: Leisure activities/psychology; Aged; Caregivers/psychology; Activities daily living/ psychology. 
BATISTA, M. P. P. et al. Repercussões do papel de cuidador. Rev. Ter. Ocup. Univ. São Paulo, v. 23, n. 2, p. 186-192, maio/ago. 2012.

\section{REFERÊNCIAS}

BARDIN, L. Análise de conteúdo. Lisboa: Edições 70, 1977.

CALDAS, C. P. Cuidador: sua instância de experiência. In: CALDAS, C. P. A saúde do idoso: a arte de cuidar. Rio de Janeiro: UERJ, 1998. p. 11-3.

IBGE - Instituto Brasileiro de Geografia e Estatística. Brasil em números: 2000. Rio de Janeiro: Fundação IBGE, 2000.

GÁlVEZ MORA, J.; et al. Perfil del cuidador principal y valoración del nivel de ansiedad y depresión. Atención Primaria, v. 31, n. 5, p. 338-339, 2003.

GRUNFELD, E.; et al. Family caregiver burden: results of a longitudinal study of breast cancer patients and their principal caregivers. Can. Med. Assoc. J., v. 170, n. 12, p. 1795-801, 2004.

KARSCH, U. M. S.; LEAL, M. G. S. Pesquisando cuidadores: visita a uma prática metodológica. In: KARSCH, U. M. S. Envelhecimento com dependência: revelando cuidadores. São Paulo: Educ, 1998. p.21-45.

LITVOC, J.; BRITO, F. C. Conceitos básicos. In: LITVOC, J.; BRITO, F. C. Envelhecimento: prevenção e promoção de saúde. São Paulo: Atheneu, 2004. p.1-16.

NERI, A. L.; CARVALHO, A. M. O bem - estar do cuidador familiar: Aspectos psicossociais. In: FREITAS, E. V.; et al. Tratado de geriatria e gerontologia. Rio de Janeiro: Guanabara Koogan, 2004. p. 778-782.

RAS VIDAL, E.; et al. Repercusiones en el cuidador de los enfermos cronicos domiciliarios. Atencion Primaria, v. 38, n. 7, p.416, 2006.

REQUIXA, R. Aspectos históricos e conceituais. In: REQUIXA, R. Sugestão de diretrizes para uma política nacional de lazer. São Paulo: SESC, 1980. p. 21-59.

RIBERTO, M.; et al. Validação da versão brasileira da medida de independência funcional. Acta Fisiatr., v. 11, n. 2, p. 72-76, 2004.

ROCHA, M. P. F; VIEIRA, M. A. V; SENA, R. R. Desvelando o cotidiano dos cuidadores informais de idosos. Rev. Bras. Enferm., v. 61, n. 6 , p. $801-808,2008$.

RODRIGUES, S. L. A.; WATANABE, H. A. W.; DERNTL, A. M. A saúde de idosos que cuidam de idosos. Rev. Esc. Enferm. USP, v. 40, n. 4, p. 493-500, 2006.

TILDEN, V. P.; et al. Out-of-hospital death: advance care planning, decedent symptoms, and caregiver burden. J. Am. Geriatr. Soc., v. 52, n. 4, p. 532-539, 2004.

WOLFF, J. L.; et al. End-of-life care: findings from a national survey of informal caregivers. Arch. Intern. Med., v.167, n. 1, p. 40-46, 2007. 